\title{
Numerical Modelling of Distributed Vibration Sensor Based on Phase-Sensitive OTDR
}

\author{
A. Masoudi* and T. P. Newson \\ Optoelectronics Research Centre, University of Southampton, Southampton, SO17 1BJ, UK \\ *Corresponding author: a.masoudi@soton.ac.uk
}

\begin{abstract}
A Distributed Vibration Sensor Based on Phase-Sensitive OTDR is numerically modeled. The advantage of modeling the building blocks of the sensor individually and combining the blocks to analyse the behavior of the sensing system is discussed. It is shown that the numerical model can accurately imitate the response of the experimental setup to dynamic perturbations a signal processing procedure similar to that used to extract the phase information from sensing setup.
\end{abstract}

Keywords: Numerical modeling; Distributed vibration sensing; Phase-sensitive OTDR.

\section{INTRODUCTION}

The volume of research on distributed optical fibre dynamic strain sensing (also known as distributed vibration sensor (DVS)) has increased substantially during the past five years. The main driving force behind this expansion was the rising demand for this type of sensors in areas such as oil and gas industry, geophysical sciences, and civil engineering. Thus far, the studies on DVSs have focused on experimentally investigating different measurement techniques [1]. To improve on the recent studies, numerical analysis of the sensing techniques investigated so far can be of significant importance [2]. The aim of this paper is to study the behaviour of the DVS proposed by Masoudi et al. [3] through numerical modelling.

\section{NUMERICAL MODELING PRINCIPLES}

The technique proposed by Masoudi et al. map dynamic perturbations along the sensing fibre based on phase-sensitive optical time domain reflectometry ( $\varphi$-OTDR). This technique uses the phase of the Rayleigh backscattered light to measure dynamic perturbations. In order to numerically analyse this technique, the backscattered electric field needs to be modeled in order to conserve the phase information of the backscattered light.

For this simulation, the modules forming the sensor such as the sensing fibre, probe pulse, and detection and acquisition units were modelled separately. This modular approach allows the modules to be modified independently which facilitates the evaluation of the effect of each individual module on the response of the sensor. For instance, the effect of the probepulse linewidth on the accuracy and resolution of the sensor can be analysed by modelling the response of the sensor to different linewidths while keeping the other elements of the simulation unchanged.

Figure 1 shows the flowchart of the numerical model used to simulate the operation of the distributed dynamic strain sensor. The model has six modules:

i. FibGen: This module was used to model the sensing fibre. It generates and randomly distribute inhomogeneities with different sizes along the length of the fibre. The inhomogeneities were used to model Rayleigh scattering;

ii. Probe Pulse: This module was used to model the probe pulse. In order to fully characterize the probe pulse, both the spatial and spectral distribution of the pulse need to be specified.

iii. Back EF: Back EF module calculates the Backscattered Electric Field detected at the front end of the fibre as a result of the interaction between the inhomogeneities along the fibre and the probe pulse. The output of the Back EF module represents the net backscattered electric field for any given spectral component in the probe pulse as a function of time from which the distance can be calculated.

iv. Strain: This module was used to model the effect of linear strain on the redistribution of the inhomogeneities along the sensing fibre. 
v. IMZI: This module computes the output the imbalanced Mach-Zehnder interferometer (IMZI) illuminated by the backscattered electric field. In this study, the MZI was assumed to comprise of a $2 \times 2$ and a $3 \times 3$ symmetrical couplers at its input and output, respectively.

vi. PD\&ADC: This module simulates the response of photodetectors (PD) and analogue-to-digital convertor (ADC) at the output of the IMZI. This module calculates the intensity of the backscattered electric field from the magnitude and phase of the backscattered electric field.

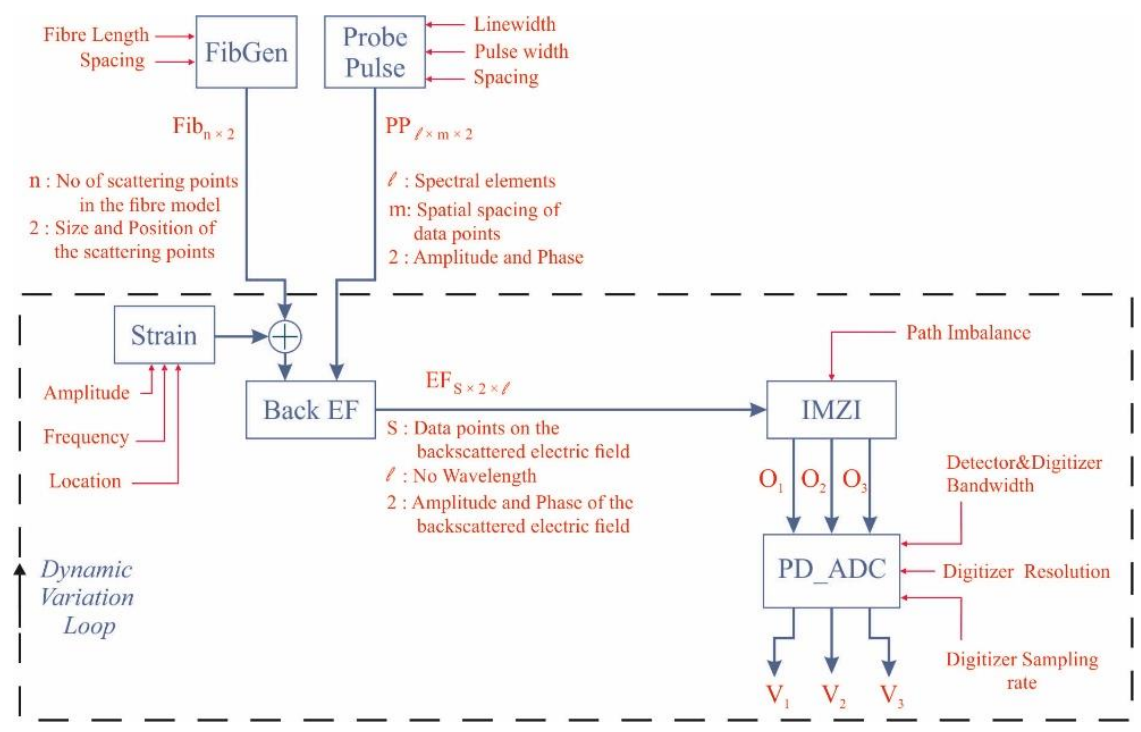

Figure 1. The flowchart of the numerical model used to simulate the operation of a distributed dynamic strain sensor.

\section{RESULTS \& DISCUSSION}

To compare the numerical model with the experimental results, the backscattered light with three different linewidths (1pm, 10pm, and 100pm) were simulated (figure 2). From the experimental results, it is known that the RMS value of the Coherent Rayleigh Noise $(\mathrm{CRN})$ is proportional to the square root of the coherence length of the light source for coherence lengths less than the length of the probe pulse:

$$
\left.V_{r m s}\right|_{C R N} \propto \sqrt{L_{\text {Coherence }}} .
$$

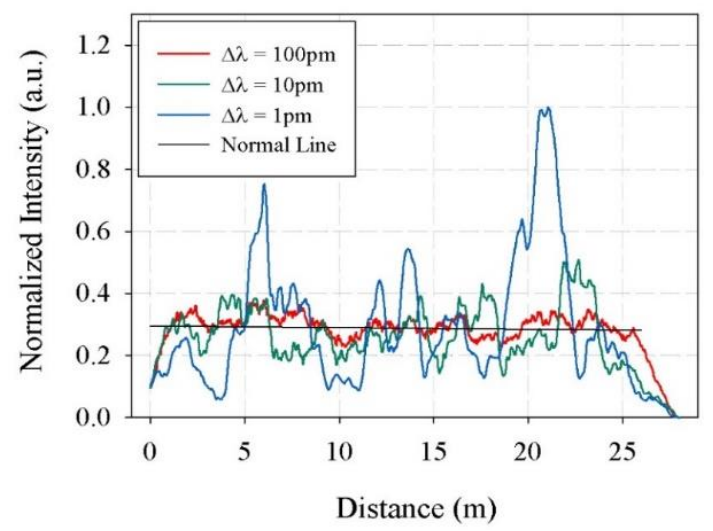

Figure 2. Coherent Rayleigh noise (CRN) on the backscattered Rayleigh traces for three different linewidths. 
Therefore, if the coherence length of a source decreases by a factor of 100, the CRN level will drop by a factor of 10 . The coherence length of light source with a linewidth of $\Delta \lambda$ is given by:

$$
L_{\text {coherence }}=\sqrt{\frac{2 \ln (2)}{n \pi}} \frac{\lambda^{2}}{\Delta \lambda}
$$

where $\lambda$ is the wavelength of the source, $\Delta \lambda$ is its linewidth, and $n$ is the refractive index of the medium. The coherence length of a 100pm light source with a central wavelength of $1550 \mathrm{~nm}$ is $16 \mathrm{~mm}$. The coherence length of a $1 \mathrm{pm}$ light source with the same central wavelength is $1.6 \mathrm{~m}$. As a result, one expect the $\mathrm{CRN}$ of a $100 \mathrm{pm}$ source to be $\sqrt{1.6 \mathrm{~m} / 16 \mathrm{~mm}}=10$ times smaller than that of a $1 \mathrm{pm}$ source. However, the coherence length of a probe pulse cannot be longer than the pulse width. Since the pulse width in the numerical model was set to be $1 \mathrm{~m}$, the coherence length of $1 \mathrm{pm}$ source is $1 \mathrm{~m}$. Consequently, the CRN of a $1 \mathrm{~m}$ pulse generated from a $100 \mathrm{pm}$ source is estimated to be $\sqrt{1 \mathrm{~m} / 16 \mathrm{~mm}}=7.9$ times smaller than the CRN of a $1 \mathrm{~m}$ pulse generated from a $1 \mathrm{pm}$ source. The simulation result shows that the CRN of 100pm is, on average, 8.13 times lower than the $\mathrm{CRN}$ of $1 \mathrm{pm}$ source. Therefore, the difference between the simulation and experimental results is less than $2 \%(7.9 / 8.13=98 \%)$. This test shows that the outcome of the numerical model agrees with the theoretical analysis as well as the experimental observations.

Similarly, experimental data shows that CRN fluctuations due to external perturbation of fibre is larger for a narrowlinewidth source compared with a source with a broader linewidth. Figure 3 shows the simulation results for $3 \mathrm{pm}$ and $30 \mathrm{pm}$ linewidth sources. The results shown in this figure agrees with the expectation from CRN of the backscattered light of a narrow and a broad linewidth sources.
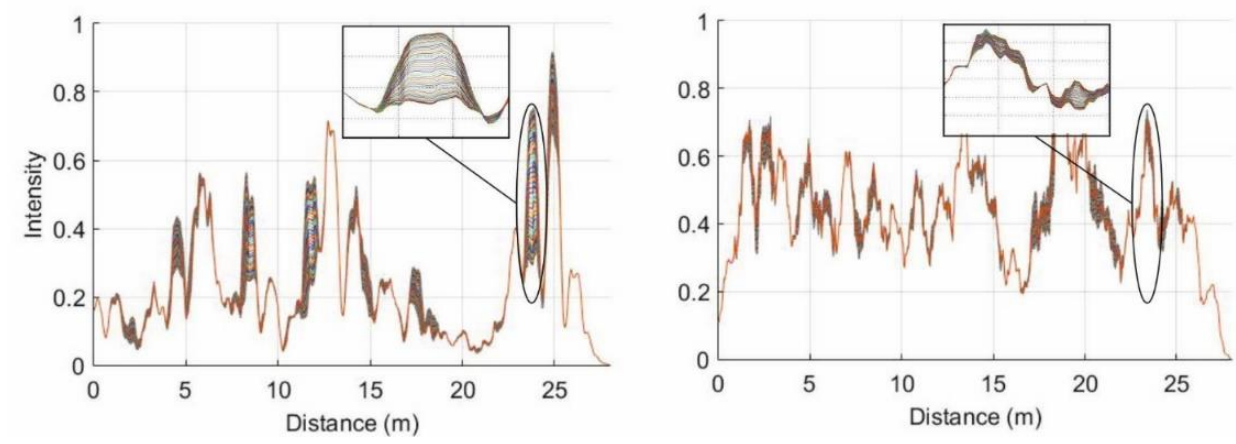

Figure 3. The simulation results of the coherent Rayleigh noise obtained by modeling a system with a) a light source with $3 \mathrm{pm}$ linewidth, and b) a light source with 30pm linewidth.

Figure 4 shows the simulation results after signal processing. The signal processing which is used to obtain this figure was identical to that used to process the data from the experimental results. For this simulation, two sinusoidal perturbations at two separate points along the fibre $(7 \mathrm{~m}$ and $18 \mathrm{~m})$ were applied. The frequency and the amplitude of the simulated strain

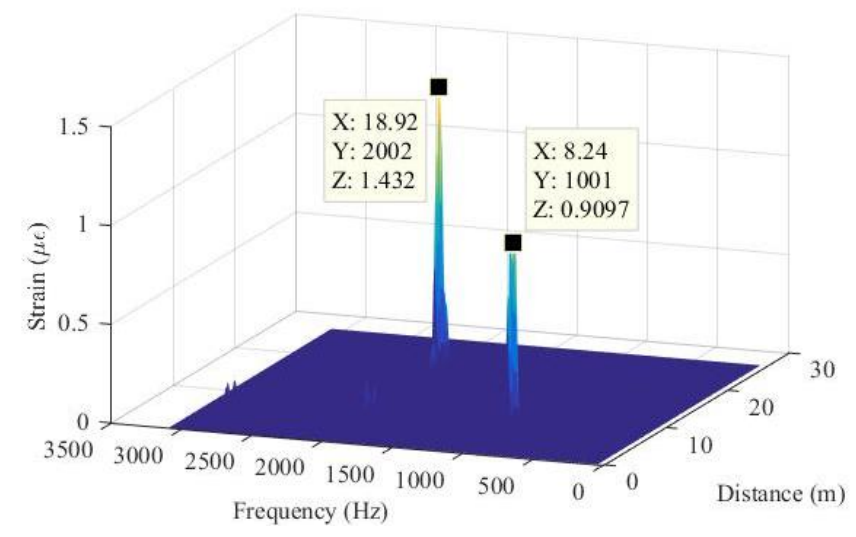

Figure 4 . The 3D plot of the simulation result post signal processing. 
at $7 \mathrm{~m}$ were $1 \mathrm{kHz}$ and $800 \mathrm{n} \varepsilon$, respectively. The frequency and the amplitude of the strain at $18 \mathrm{~m}$ point were $2 \mathrm{kHz}$ and $1.5 \mu \varepsilon$, respectively. The $3 \mathrm{D}$ plot of figure 4 shows two peaks, their locations, frequencies and amplitudes. The two peaks correctly indicate the characteristics of the two perturbations at the two locations on the fibre.

Figure 5 shows the simulation results for different strain rates. The data points and their uncertainty ranges were obtained by modelling the effect of ten sinusoidal perturbations imposing the same frequency an amplitude at different positions along the fibre. The circles in this plot show the average value of the strains while the error bar at each data point indicates the standard deviation of the results. The uncertainty in the strain measurement is the direct result of the randomness in the distribution of inhomogeneities in the fibre. Although the phase difference between any two sections of the fibre varies linearly with the induced strain between them, the phase of the backscattered light from those sections vary randomly with applied strain. This random variation gives rise to nonlinearity between the induced and measured strain. Figure 5 shows that the outcome of the numerical model is consistent with the experimental results i.e. both sets of results demonstrate that the output of the sensor has a linear relationship with the imposed strain on the sensing fibre.

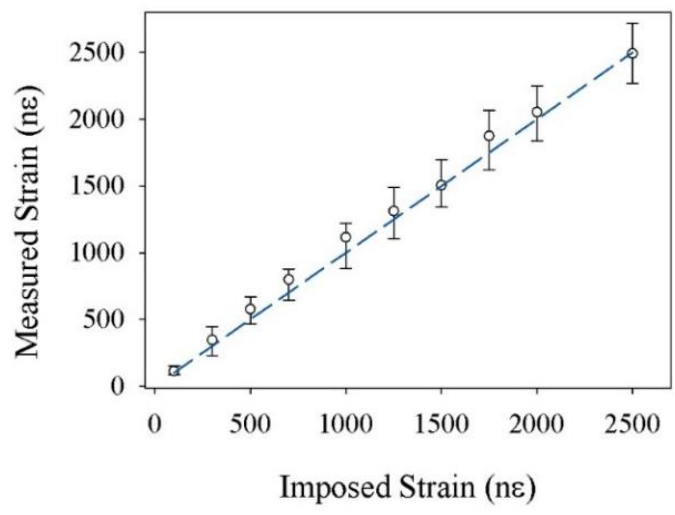

Figure 5. Linearity of the simulation results.

\section{CONCLUSION}

In conclusion, a distributed vibration sensor based on $\varphi$-OTDR was numerically modeled. It was argued that to increase the flexibility of the model, it is best to break the sensing system into its fundamental building blocks, model each block separately, and then combining the blocks to assess the behavior of the sensor. Simulation results showed that the mathematical model can accurately simulate the response of a DVS system to dynamic perturbations. The model can later be used for noise and linearity analysis of such systems.

\section{ACKNOWLEDGEMENT}

This work is funded by EPSRC UK grant EP/N00437X/1.

\section{REFERENCES}

[1] A. M. a. T. P. Newson, "Contributed Review: Distributed optical fibre dynamic strain sensing," Review of Scientific Instrument, vol. 87, p. 011501, 2016.

[2] L. B. L. e. al., "Fundamentals of Optical Fiber Sensing Schemes Based on Coherent Optical Time Domain Reflectometry: Signal Model Under Static Fiber Conditions," Journal of Lightwave Technology, vol. 33, pp. 3660-3671, 2015.

[3] M. B. a. T. P. N. A. Masoudi, “A distributed optical fibre dynamic strain sensor based on phase-OTDR,” Measurement, Science and Technology, vol. 24, p. 085204, 2013. 